\title{
Evaluación del programa Todos a Aprender: Resultados de la evaluación de contexto
}

\author{
Program evaluation Todos a Aprender: Evaluation's results of context
}

\author{
Sandra Milena Díaz*, Carlos Barreira**, Maria do Rosario Pinheiro** \\ *Doctoranda Facultad de Psicología y Ciencias de la Educación, Universidad de Coimbra.** Profesores Facultad de Psicología y \\ Ciencias de la Educación, Universidad de Coimbra,
}

\begin{abstract}
Resumen
Este artículo presenta resultados preliminares de la evaluación multifactorial del programa Todos a Aprender (PTA), para la transformación de la calidad, implementado en Colombia desde 2011, para mejorar los aprendizajes de los estudiantes de la básica primaria. Dicha evaluación se fundamenta en un marco teórico que focaliza los conceptos de calidad y evaluación y se desenvuelve a la luz de los métodos mixtos de investigación. Los resultados de esta primera fase, evaluación de contexto, logrados mediante análisis documental, indican que el programa resulta pertinente para responder a las principales necesidades identificadas tanto en la realidad nacional como en el ámbito latinoamericano.
\end{abstract}

Palabras clave: evaluación de contexto, programa "Todos a Aprender”, calidad educativa.

\begin{abstract}
The current article provides preliminary results of the multifactorial evaluation of the Todos a Aprender program to enhance educational quality; it has been implemented since 2011 in Colombia in order to improve the learning of students at elementary school. The evaluation is supported by a theoretical framework that focuses on the concepts of quality and evaluation and it is developed through mixed methods research. The results of the first phase, evaluation of context, achieved by documental analysis indicate that the program is relevant to meeting the main needs identified in both the national situation as well as the Latin American context.
\end{abstract}

Keywords: evaluation of context, "Todos a Aprender" program, educational quality.

\section{El PTA: surgimiento y estructura}

El programa Todos a Aprender (PTA) es una propuesta diseñada e implementada por el Ministerio de Educación Nacional de Colombia para mejorar los aprendizajes de los estudiantes de la educación básica primaria en las áreas de lengua española y matemáticas; especialmente en las instituciones focalizadas por presentar bajos desempeños, de acuerdo con los resultados de las pruebas nacionales de evaluación, pruebas Saber (ICFES, 2010). De esta forma, surge en el marco de la política educativa del periodo 2011-2014 cuyo principal interés es garantizar la calidad educativa como estrategia para alcanzar la prosperidad.

Mediante un sistema que integra diferentes aspectos asociados a la calidad y que focaliza la formación de los profesores como la principal apuesta para el mejoramiento de las prácticas de aula, el programa integra cinco componentes que se articulan para lograr una atención integral a las instituciones (figura 1): El componente pedagógico, asociado con los referentes curriculares nacionales y la evaluación de aprendizajes; el componente de formación situada, encargado de la formación y acompañamiento a los docentes de aula; el componente de gestión educativa, que busca la formación de los directivos docentes y la integración del programa como parte del plan de mejoramiento de cada institución educativa; el componente de infraestructura, que da cuenta de aspectos relacionados con el acceso y la permanencia de los estudiantes en las escuelas; y por último un componente transversal que busca la socialización del programa con la comunidad y su movilización en función de los objetivos establecidos.

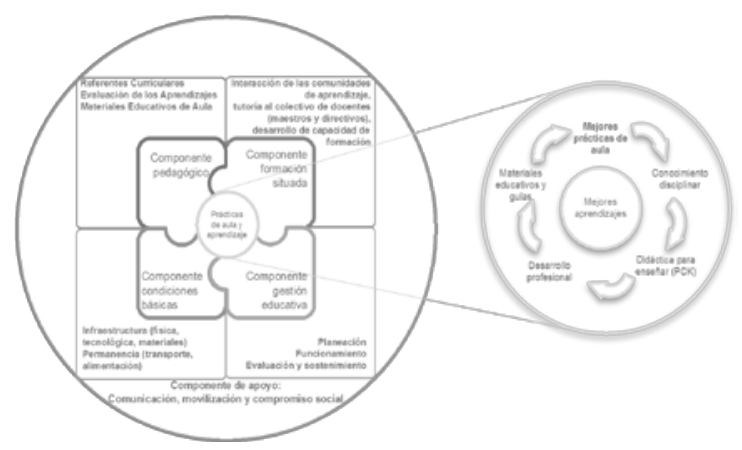

Figura 1. Visión sistémica integral de la estrategia del PTA. (Fuente: MEN, 2013. Guía 1. Sustentos del Programa, p. 11)

La estrategia de operación del programa funciona através de la formación en cadena. El primer eslabón corresponde a la gerencia encargada de la administración general, luego encontramos un equipo de coordinadores de cada uno de los componentes y un ejercito de 100 formadores que acompaña a 3000 tutores, quienes a su vez tienen la misión de desarrollar procesos de formación in situ dirigidos a los docentes de las instituciones educativas focalizadas.

\section{La calidad en la educación: concepto polivalente}

En su constante búsqueda por mejorar el sistema, las políticas educativas de muchos países del mundo han atendido temas estructurales como la cobertura y la calidad. En efecto, según el planteamiento de Martinic (2008), podemos ver la progresión de las recientes 
reformas educativas en función de tres generaciones clave, la de los ochenta que apuntaba esencialmente a la gestión, financiamiento y acceso al sistema; la de los noventa, cuyo foco de atención se direccionó hacia la calidad de los procesos y resultados del sistema educativo, y las de inicio del siglo XXI, que encaminaron la transformación desde un paradigma de resultados, más que de estrategias, y de fuertes liderazgos.

No obstante, estos tres momentos no se suceden de forma claramente delimitada y en muchos casos, aparecen simultáneamente en el complejo panorama educativo, lo que permite evidenciar múltiples comprensiones de los fines del sistema, los procesos pedagógicos y especialmente, el concepto mismo de calidad y los factores que permiten explicarla.

Esta variedad de aproximaciones a la calidad se ve legitimada por su carácter dinámico y contextualizado; de modo tal que establecer un concepto limitado y único nos llevaría a desconocer el consenso sobre su carácter polisémico, polivalente, social, político y en definitiva integral, ya apuntado por diversos autores (Gallego \& Rodríguez, 2014; González, 2000; Valdés, 2008).

La calidad, desde una perspectiva instrumental, puede estar asociada a la noción de efectividad, especialmente en el paradigma proceso-producto en el que la efectividad del profesor influye de manera decisiva en los resultados de los estudiantes (WB, 2011). Por otro lado, desde una perspectiva social y de derechos, la calidad se encuentra estrechamente vinculada a procesos de transformación y responsabilidad social (Martínez, 2014).

En esta segunda perspectiva, encontramos posiciones como la de UNESCO (2004) que asume la calidad como una combinación de condiciones, entre las que se destacan las de orden social, para la enseñanza aprendizaje y el logro académico de los alumnos; lo cual queda reforzado con la visión integral propuesta por OREALC-UNESCO (2007), en la que se subrayan cinco dimensiones integradas en la calidad: equidad, relevancia, pertinencia, eficacia y eficiencia. La definición propuesta por algunos autores ha estado circundando esta integralidad del concepto desde el realce dado a algunas de estas dimensiones: equidad e igualdad de oportunidades (Gallego \& Rodríguez, 2014), oportunidad y pertinencia (Bralavsky, 2006).

Una vez asumida esta amplitud en el concepto y en aras de llegar a algún nivel de operacionalización, los intentos por identificar factores concretos asociados a la calidad, no han sido pocos. Gran parte de ellos coinciden en destacar la calidad de los docentes y por tanto su permanente formación (Marchesi, 2010; Mckinsey 2007; UNESCO, 2004; UNICEF, 2010), igualmente se subraya la importancia de aspectos de orden pedagógico, como la calidad del currículo y del material de aprendizaje (UNICEF, 2010); así como aspectos de orden físico para garantizar adecuados espacios de aprendizaje (Marchesi, 2010, UNESCO, 2004;) y finalmente el liderazgo escolar (Bralavsky, 2006; Mckinsey, 2007; OCDE, 2012; UNESCO, 2004). La articulación entre la escuela y la familia, también se apunta como factor determinante en el buen desempeño de los estudiantes (UNICEF, 2010).
En cualquier caso, y con la salvedad de la difícil empresa que comporta el avanzar en la definición de calidad, sus dimensiones y aspectos determinantes, consideramos que ésta, en nuestro contexto particular, debe ser una educación que garantice aprendizajes básicos tanto en términos cognitivos como de promoción de valores; que busque la promoción de la equidad con un sentido de pertinencia en un contexto social con marcadas diferencias; que garantice docentes formados y con el reconocimiento justo para gestionar ambientes de aprendizaje adecuados; que proporcione condiciones básicas que permitan los aprendizajes y finalmente que articule diferentes estrategias, acciones y actores sociales, es decir no solamente profesores y alumnos. Todo esto sumado a un uso eficiente de tiempos y recursos, entendiendo la eficiencia no en una lógica económica, sino en una lógica pedagógica, como lo resaltan Aguerrondo \& Xifra (2002).

\section{La evaluación de programas: aproximaciones teóricas}

Establecer las estrategias y procedimientos para evaluar un programa que se inserta en el marco de una política de calidad, requiere no solo la comprensión del concepto de calidad como meta del sistema, sino también como finalidad de la evaluación (Stake, 2006). En consecuencia, es preciso analizar también las características de un buen programa y lo que su valoración implica.

Un programa se asocia a tres elementos fundamentales, una acción intencionada que persigue unas determinadas finalidades e interviene en un contexto social específico para procurar la promoción educativa de las personas a quien va dirigido, además debe estar sistemáticamente diseñado partiendo de la definición de unos objetivos precisos y realistas, contar con recursos en cantidad y calidad ajustado a las metas, y finalmente, ser implementado según su planeación (Gobantes, 2000).

Ahora bien, en estas condiciones ideales es importante considerar dos aspectos clave para diseñar la evaluación del programa: el grado de complejidad (extensión geográfica, cantidad y diversidad de acciones) y la impronta del contexto social, político, administrativo y económico en que se desarrolla y de las personas involucradas en él. Por tanto, es la evaluación la que debe adaptarse al programa y de ahí que se requiera un diseño específico según su propósito y dimensiones.

En este sentido, asumimos la evaluación como un concepto complejo y multidimensional, en constante construcción (Guba \& Lincoln, 1989) y por tanto, recogemos diferentes perspectivas que contribuyen a nuestra visión general y a conformar un diseño adecuado al objeto que nos ocupa. El establecimiento de un referencial claro para determinar el valor o mérito de un objeto es quizá la primera condición asociada al concepto de evaluación (Fitzpatrick, Sanders \& Worthen 2004; Guba \& Linconl, 1989; Scriven, 1991); además de la identificación del valor en sí mismo, es importante la determinación de su impacto y en consecuencia sus posibilidades de mejora, mediante un proceso de toma de decisiones (Stufflebeam \& Shinkfield, 1993). 
La necesaria sistematicidad en el proceso, la ineludible condición de implementar criterios y la toma de decisiones de mejora, también han sido señaladas por (Pérez, 2014); asímismo, Stake (2006) distingue dos formas posibles de evaluación subrayando que ambas deben "estar incluidas en los estudios de calidad de programas, aun cuando no sean fáciles de combinar, e incluso, se contradigan entre si” (p. 17), se trata de la evaluación basada en estándares (medición) y la evaluación enfocada en la experiencia (evaluación comprensiva), con lo que además de valores o méritos destaca la comprensión de significados por parte de los stakeholders.

Asociados a estos principios se han propuesto diferentes modelos de evaluación que van desde la comparación explícita objetivos-resultados (modelo tyleriano), hasta una evaluación más integral y comprensiva (Stake, 2006) que defiende la necesidad de tomar en consideración los antecedentes del objeto de evaluación y las operaciones desarrolladas.

Estas dos consideraciones son complementarias al modelo propuesto por Stufflebeam y Shinkfield (1993), el modelo CIPP (context, input, process, product) que destaca, como etapas fundamentales de la evaluación: la definición del contexto, en términos de población y necesidades básicas; la valoración de la capacidad del programa en términos de estrategias y presupuestos; la evaluación de los procesos implementados, y finalmente la evaluación los resultados.

Así pues, aunque dicho modelo se convierte en nuestro punto de referencia para estructurar el proceso de evaluación, también aprovechamos las contribuciones de la propuesta de Stake (2006) que además sugiere establecer las intenciones (lo planeado) frente a las observaciones (lo percibido) y las normas (convicción personal frente a lo bueno y deseable), partiendo de la identificación de la base lógica del programa. Igualmente se destaca su constante interés por hacer partícipe los diferentes participantes en el proceso.

Igualmente, Scriven (1991) ofrece algunas claves, al señalar la necesidad de hacer una evaluación intrínseca, es decir de la cualidad y procedimientos del objeto o mediación (lo que implica dar cuenta de la estructura el programa) y una evaluación extrínseca que ya no se preocupa de la naturaleza del programa sino del efecto causado; con lo que propone un multimodelo de evaluación, que parte de preguntas básicas como: ¿Qué hay que evaluar? ¿Cuáles son sus componentes?, ¿Cuáles son los antecedentes y el contexto? Todas estas contribuciones resultan fundamental en nuestro diseño.

\section{Marco Metodológico: los métodos mixtos}

De acuerdo con la necesidad de diseñar un modelo ajustado a la naturaleza del objeto evaluado y al objetivo general del proceso de evaluación que en este caso es evaluar el PTA en el contexto colombiano, identificando su valor intrínseco (en términos de estructura y desarrollo) y su valor extrínseco o coherencia interna (en función de la pertinencia con el contexto y los resultados logrados) desde el marco de la calidad educativa, optamos por acoger los métodos mixtos de evaluación como marco metodológico.
Nos encontramos entonces con lo que Pérez (2014) cataloga como el principio de complementariedad metodológica y Fernándes (2010) describe como “a tentativa de desenvolver uma perspectiva mais pragmática, conjugando sobretudo metodologías, que evita submeter-se a qualquier ortodoxia epistemológica, ontológica ou metodológica” (p. 23). En efecto, los métodos mixtos de investigación se constituyen en una opción que nos permite mezclar o combinar técnicas y métodos cualitativos y cuantitativos en un solo estudio (Onwuegbuzie \& Johnson 2004).

Esta tendencia de integración metodológica es respaldada por diversos autores y se encuentra estrechamente ligada a la evaluación educativa (Díaz, 2014; Fitzpatrick, Sanders \& Worthen, 2004; Greene, 2013). Específicamente en lo que a evaluación de programas corresponde, Cook \& Reichardt (2005), señalan que es probable que la descripción del contexto y el grado en que se desarrolla el programa se realice con mayor eficacia conforme a un método cualitativo, que la valoración del impacto se realice con mayor precisión mediante métodos cuantitativos y que la explicación causal ( proceso por el cual se alcanzó el efecto logrado) se obtenga de mejor manera a través del empleo conjunto de métodos cuantitativos y cualitativos.

El diseño particular seleccionado para nuestro propósito en el marco de esta metodología es el diseño de triangulación, que nos permite recolectar y analizar datos de forma independiente utilizando técnicas tradicionales asociadas a cada metodología específica. En el marco de este diseño la variante que consideramos más adecuada es el multinivel model en el que "different methods (quantitative and qualitative) are used to address different levels within a system. The findings from each level are merged together into one overall interpretation" (Creswell \& Plano, 2007, p. 65).

Así las cosas, desarrollamos la investigación en cuatro fases, evaluación de contexto, en la que apelamos a una técnica de análisis documental, la evaluación de diseño, donde recurrimos a la misma técnica, la evaluación del proceso en la que hemos diseñado y aplicado entrevistas y cuestionarios a los coordinadores del programa y a los formadores, tutores y profesores respectivamente; y por último la evaluación de resultados, en la que combinamos técnicas cualitativas y cuantitativas. En esta última, mediante un estudio comparativo-causal, determinamos los logros de las instituciones educativas en relación con las pruebas Saber y gracias a un estudio cualitativo (análisis de contenido) identificamos logros no previstos asociados a experiencias significativas.

Los resultados que presentamos en este artículo corresponden a los hallazgos de la primera fase de evaluación obtenidos mediante un estudio documental. En relación con las fuentes, específicamente se recurrió al análisis de informes de investigación y otros documentos que dan cuenta de la situación educativa tanto del país como de este en el contexto latinoamericano, con el ánimo de tener una perspectiva general que no se reduzca al contexto interno. Esta valoración de las necesidades, problemas fortalezas y debilidades se constituye en la primera etapa de todo proceso de evaluación de programas (Pérez, 2014). 


\section{Evaluación de contexto: resultados y discusión}

De acuerdo con la situación expuesta a partir de la revisión documental y en función del objetivo que orientó esta primera fase de evaluación, es decir identificar el grado de pertinencia entre el PTA frente a las necesidades y problemáticas del contexto colombiano podemos afirmar que se evidencia la pertinencia del programa, dado que dentro de las principales necesidades resaltadas en los diferentes estudios a nivel lationamericano (Poggi, 2014; UNESCO, 2013) y en el ámbito nacional (Barrera, Maldonado \& Rodríguez, 2012; ICFES, 2010) figura el fortalecimiento del aprendizaje de los estudiantes en las áreas básicas y especialmente en las poblaciones más vulnerables, por su condición de ruralidad o bajo estrato socioeconómico.

$\mathrm{Si}$ atendemos a factores de corte cuantitativo, vemos que tanto las pruebas estandarizadas internacionales (PISA, 2009; SERCE, 2006) como los resultados reportados en las pruebas nacionales (Saber, 2009), evidencian que el nivel de desarrollo de las competencias de los estudiantes colombianos ocupa los niveles más bajos y por tanto, no logra ser garante ni de desempeños básicos ante las exigencias de este siglo, ni de aprendizajes posteriores. En consecuencia, una intervención para mejorar estos resultados, resulta pertinente; y de hecho para el PTA, dichos datos fueron asumidos como línea de base, según se advierte en su guía de sustentos. (MEN, 2013)

Además de estas carencias explícitas relacionadas con los estudiantes, se torna inaplazable la necesidad de formación docente, especialmente en las zonas más carenciadas, en lo que también coindicen tanto los análisis internacionales (OEI, 2010; UNESCO, 2013) como los resultados de investigaciones internas (García, Maldonado \& Rodríguez, 2014). Al ser este el foco del programa, parece que la prioridad dada a esta necesidad dentro del contexto está justificada, lo que además concuerda con todas las propuestas que señalan la formación de los docentes como factor clave para la transformación de la calidad. Recordemos que "las necesidades que dan lugar a programas pueden ser detectadas en las personas o en los grupos para los que se diseñan los programas, pero, también, pueden responder a carencias más o menos generales de la sociedad que, para bien, deberían ser atendidas mediante la formación y capacitación de las personas” (Pérez, 2014, p. 169).

Cabe resaltar además que las condiciones de los docentes y las disposiciones legales internas en relación con la regulación de su profesión parecen reforzar la necesidad de ofrecer un programa que contribuya a la cualificación permanente, lo que además se articula con el sistema de evaluación de desempeño en el que el docente debe dar cuenta de un conjunto de conocimientos de orden disciplinar y didáctico. No obstante, en el PTA no se hace explícita alguna referencia a la revisión de esta prueba como línea de base de la formación docente, probablemente dado que esta se implementó realmente solo en el año 2010, aunque, a nuestro juicio, debería ser un instrumento para ser aprovechado por el programa, no solo en la selección de los tutores, sino además en el reconocimiento de los desafíos en relación con la formación de los docentes.

Otros de los factores que, en la caracterización del contexto, son resaltados como necesidades básicas a ser atendidas tiene que ver con el aseguramiento de insumos o recursos, tanto desde el punto de vista pedagógico como desde el punto de vista físico, es decir infraestructura (Barrera, 2014; Barrera et al. 2012; OEI, 2010, UNESCO, 2003), con lo que la atención a estos aspectos por parte del PTA desde los componentes pedagógico y de condiciones básicas, respectivamente, podría subsanar estas deficiencias.

Además de estos aspectos, la gestión institucional de calidad se convierte en un requerimiento básico en el contexto internacional (UNESCO, 2013) y nacional (Barrera, 2014), por lo que la apuesta del programa por la formación de directivos docentes en el marco del componente de gestión, podría resultar adecuada a la demanda expuesta.

La revisión de los referentes curriculares es otro de los elementos en causa en el ámbito internacional, no obstante parece no ser un llamado insistente en los estudios nacionales. En cualquier caso, estos referentes se asumen como un insumo del programa dentro del componente pedagógico y no se focaliza su revisión, sino su aprovechamiento para orientar tanto los materiales educativos como su apropiación para diseñar las didácticas, a nuestro juicio sería importante someterlos a procesos de revisión y actualización.

Finalmente, emergen como aspectos a ser atendidos el fortalecimiento de las relaciones entre docentes, alumnos y padres, y el papel que debe darse a la formación ciudadana y al uso de las nuevas tecnologías. Estos tres factores no tienen visibilidad en el programa, dado que se han priorizado otros elementos, cuyo tratamiento parece ser más apremiante en aras de garantizar la base sobre la cual empezar a trabajar otros aprendizajes. Además, el Ministerio cuenta con otros programas que apuntan a la calidad no solo en función de lo cognitivo sino también de lo actitudinal.

En suma, la valoración de este primer nivel nos indica que se trata de un programa que atiende a características básicas como el reconocimiento del contexto social específico en el que se inserta, comprende dimensiones clave de la calidad como la equidad, relevancia y pertinencia; y finalmente, da cuenta de aspectos generalmente ligados a la calidad; por lo que su relevancia es evidente en el contexto en que emerge.

\section{Referencias}

Aguerrondo, I., \& Xifra, S. (2002). La escuela del Futuro. Cómo piensan las escuelas que innovan. Papers Editores: Buenos Aires.

Barrera, F., Maldonado, D. \& Rodríguez, C. (2012). Calidad de la educación básica y media en Colombia: Diagnóstico y propuestas. Serie Documentos de trabajo No. 126. Universidad del Rosario. Facultad de Economía.

Barrera, M. (2014). La Educación Básica y media en Colombia: Retos en equidad y Calidad. Informe Final. Bogotá: Fedesarrollo 
Bralavsky, C. (2006). Diez factores para una educación de calidad para todos en el siglo XXI. Revista electrónica iberoamericana sobre calidad, eficiencia y cambio en educación. 4(2e), 84-101. Consultado en agosto 2014 en http://www.rinace.net/arts/vol4num2e/art5.pdf.

Cook, T., \& Reichardt, S. (2005). Métodos cualitativos y cuantitativos en investigación evaluativa. ( $\left.5^{\mathrm{a}} \mathrm{ed}\right)$. Madrid: Ediciones Morata.

Creswell, J., \& Plano, V. (2007). Designing and Conducting Mixed Methods Research. California: Sage publications, Inc

Díaz, S. (2014). Los Métodos Mixtos de Investigación: Presupuestos generales y aportes a la evaluación educativa. Revista Portuguesa de Pedagogía. 48(1), 7-23.

Fernandes, D. (2010). Acerca da articulação de perspetivas e da construção teórica em avaliação educacacional. En: M. Esteban \& A. Afonso. Olhares e interfaces. Reflexões críticas sobre a avaliação. (pp. 15- 44). Sao Paulo: Cortez Editora.

Fitzpatrick, J., Sanders, J., \& Worthen, B. (2004). Program evaluation. Alternative approaches and practical guidelines. ( $3^{\mathrm{a}}$ ed.). Boston: Pearson Education

Gallego, J. \& Rodríguez, A. (2014). El reto de una educación de calidad en la escuela inclusiva. Revista Portuguesa de Pedagogía. 48(1), 39-54.

García, S., Maldonado, D., Perry, G., Rodrígues, C \& Saavedra, J. (2014) Tras la excelencia docente. Cómo mejorar la calidad de la Educación para todos los colombianos. Bogotá: Fundación Compartir

Gobantes, J. ( 2000). Calidad y evaluación de programas: Usos y diseño de la evaluación. En: T. González. Evaluación y gestión de la calidad educativa un enfoque metodológico (pp 83-125). Málaga: Ediciones Aljibe.

González, T. (2000). Evaluación y gestión de la calidad educativa. En: Evaluación y gestión de la calidad educativa, un enfoque metodológico (49-79). Málaga: Ediciones Aljibe.

Greene, J. (2013). The educative evaluator. In M. Alkin (Ed.), Evaluation Roots (pp. 97-105). California: Sage Publication.

Guba, E., \& Lincoln, Y. (1989). Fourth generation evaluation. California: Sage Publication.

Instituto Colombiano para el Fomento de la Educación Superior- ICFES-. (2010). Informe resultados Nacionales. Saber $5^{\circ}$ y $9^{\circ}$. Bogotá.

Marchesi, A. (2010). Estrategias para el cambio educativo. En: A. Marchesi \& M. Poggi (coord.). Presente y futuro de la Educación Iberoamericana. (pp.91-11). Agencia Española de Cooperación Internacional para el desarrollo y Fundación Carolina.

Martínez, F. (2014). Alternativas para evaluar la calidad social de la educación. Revista iberoamericana de Educación. 68(2), 1-14.

Martinic, S. (2008). Información, participación y enfoque de derechos. En: UNESCO \& LLECE, Reflexiones en torno a la evaluación de la calidad educativa en América Latina y el Caribe. (pp.13-34) Santiago de Chile: Salesianos impresores

McKinsey \& Company (2007). How the World's Best-Performing School Systems Come Out On Top. Consultado en: marzo de 2014, en http://mckinseyonsociety.com/how-the-worlds-best -performing-schools-come-out-on-top/

Ministerio de Educación Nacional de Colombia (MEN) (2013). Programa todos a aprender para la transformación de la calidad educativa. Guía 1. Sustentos del programa. Consultado en febrero 2013, en:http://www.mineducacion.gov.co/1621/articles310 69_archivo_pdf_sustentos_junio27_2013.pdf

OCDE. (2012). Equity and Quality in Education: Supporting Disadvantaged Students and Schools. Consultado en abril 2015 en http://dx.doi.org/10.1787/9789264130852-en

Onwuegbuzie, A., \& Johnson, B. (2004). Mixed Methods Research: A Research Paradigm Whose Time Has Come. Educational Researcher, 33(7) 14-26. Consultado en diciembre 2013, en http://www.aera.net

OREALC-UNESCO.(2007). El derecho a una educación de calidad para todos en América Latina y el Caribe. Revista electrónica Iberoamericana sobre calidad, Eficiencia y Cambio en Educación. 5 (3), 1-21.

Organización de Estados Iberoamericanos para la Educación, la Ciencia y la Cultura. (OEI) (2010). Metas educativas 2021. La educación que queremos para la generación de los Bicentenarios. Madrid: autor.

Pérez, R. (2014). Evaluación de programas Educativos. ( $2^{\text {a }}$ ed.). Madrid: Editorial la Muralla.

Poggi, M. (2014). La Educación en América Latina: logros y desafíos pendientes: documento básico. Buenos Aires: Santillana.

Scriven, M. (1991). Evaluation Thesaurus (4 $4^{\text {a }}$ ed.). California: Sage Publication

Stake, R. (2006). Evaluación comprensiva y evaluación basada en estándares. Editorial Grao: Barcelona.

Stufflebeam, D., \& Shinkfield, A. (1993). Evaluación sistemática. Madrid: Paidos.

UNESCO (2004). Educación para todos el imperativo de la calidad. Informe de seguimiento de la EPT en el mundo. Paris: Graphoprint

UNESCO (2013). Situación Educativa de América Latina y el Caribe: Hacia la Educación de Calidad para todos al 2015. Santiago: Oficina Regional de Educación para América Latina y el Caribe.

UNICEF (2010). Defining Quality in Education. Working paper Series. Education Section. New York.

Valdés, H. (2008). Hacia una evaluación del desarrollo, formación corporal, racional y emocional del ser humano. El caso cubano. Reflexiones en torno a la evaluación de la calidad educativa en América Latina y el Caribe. (pp.67-90) Santiago de Chile: Salesianos impresores.

World Bank (2011). Building better teachers in the Caribbean. Consultado en marzo de 2014 en:http://siteresources.worldbank.org/EDUCATION/Re sources/WB-Bruns_BuildingBetterTeachersintheCaribb ean-1.pdfo 